\title{
Pursuing an underdiagnosed disease: a simple imaging test for increasing suspicion of cardiac amyloidosis
}

\author{
Rodney H. Falk • Sharmila Dorbala
}

Published online: 26 January 2011

(C) Springer-Verlag 2011

The systemic amyloidoses are a group of uncommon diseases in which the common factor is infiltration of one or more organs with a noncellular, extracellular proteinaceous material with specific light microscopic staining characteristics [1]. Significant advances in treatment have been made over the past decade, particularly in $\mathrm{AL}$ amyloidosis in which the precursor protein is derived from immunoglobulin light chain fragments [2]. Transthyretin (TTR)-derived amyloidosis has also been the subject of intensive investigation, and several new therapeutic approaches have completed, or are approaching, preliminary clinical testing [3-7]. Refinements in immunohistochemical and proteomic techniques have permitted precise definition of the amyloid type, a prerequisite for initiating appropriate (and avoiding inappropriate) therapies [8].

In 2005, the Bologna group published data from 25 patients with amyloidosis (17 of whom had TTR-related amyloidosis) suggesting the bone-scanning agent ${ }^{99 \mathrm{~m}} \mathrm{Tc}-$ 3,3-diphosphono-1,2-propanodicarboxylic acid (DPD) was preferentially taken up by the heart in patients with TTR amyloidosis, whereas it showed no uptake in AL amyloid-

R. H. Falk $\cdot$ S. Dorbala

Cardiac Amyloidosis Program, Brigham and Women's Hospital,

Harvard Medical School,

Boston, MA, USA

\section{S. Dorbala}

Section of Nuclear Cardiology, Brigham and Women's Hospital, Harvard Medical School,

Boston, MA, USA

\section{R. H. Falk $(\bowtie)$}

Harvard Vanguard Medical Associates,

133, Brookline Ave,

Boston, MA 02215, USA

e-mail: rfalk@partners.org osis or in controls without the disease [9]. This appeared to be isotope specific, as ${ }^{99 \mathrm{~m}} \mathrm{Tc}-\mathrm{methylene}$ diphosphonate (MDP) showed no uptake in 11 patients who showed distinct myocardial uptake with ${ }^{99 \mathrm{~m}} \mathrm{Tc}-\mathrm{DPD}$. The authors have now expanded their original observations and, while confirming the tracer is avidly taken up in the TTR amyloid patients, they now also describe a mild to moderate uptake in one third of AL patients, thereby reducing the specificity of the test for TTR amyloidosis alone but still rendering it an interesting and informative technique [10].

Uptake of technetium-based bone imaging traces by the heart in cardiac amyloidosis is not a newly described phenomenon. As pointed out by Rapezzi and coauthors and recently reviewed in detail [11], several other nuclear imaging modalities have been proposed for imaging cardiac amyloidosis, such as ${ }^{99 \mathrm{~m}} \mathrm{Tc}$-aprotinin [12] and ${ }^{123}$ I-metaiodobenzylguanidine (MIBG) [13]. Over 25 years ago the uptake of technetium pyrophosphate (then used as a myocardial infarction imaging agent) was described in both $\mathrm{AL}$ and TTR amyloidosis, although the sensitivity of the imaging was subject to debate [14-16]. The type of technetium-based isotope was believed to be significant, as ${ }^{99 \mathrm{~m}} \mathrm{Tc}-\mathrm{MDP}$ showed little consistency for imaging amyloid cardiomyopathy, despite occasional case reports of cardiac uptake [17]. With the rapid advances in echocardiography and the echocardiographic descriptions of amyloid cardiomyopathy, technetium pyrophosphate cardiac imaging for possible amyloid cardiomyopathy became obsolete, limited to a radiologic curiosity when incidentally seen on a scan performed for other reasons such as for the evaluation of metastatic disease. Echocardiography still remains the mainstay for the initial suspicion/diagnosis of cardiac amyloidosis [1] (and was also a component of the "gold standard" used in this study), and is nowadays often complemented by cardiac MRI which 
demonstrate several highly specific features in this disease regardless of the precursor protein $[18,19]$.

The apparent preference of ${ }^{99} \mathrm{~m} \mathrm{Tc}-\mathrm{DPD}$ for TTR amyloidosis over $\mathrm{AL}$ amyloidosis for imaging cardiac involvement is intriguing but the reason is unclear. There were some AL patients with cardiac uptake and they were found to have a slightly lower serum calcium level compared to those without uptake. The investigators raise the possibility that this might be related to a particularly high calcium concentration in the heart in this subgroup, due to the avidity of amyloid fibrils for the binding of calcium. While the differences in serum calcium were small, and may have been a chance finding, we suggest a possible alternative explanation. Unlike TTR amyloidosis, $\mathrm{AL}$ amyloidosis is frequently associated with heavy proteinuria and associated hypoalbuminemia. This will be associated with low serum calcium. It may be that there is more available isotope in patients with hypoalbuminemia, due to altered regional extracellular fluid dynamics and tracer kinetics [20]. Unfortunately serum albumin levels are not documented, but it would be of interest to address this, particularly as hypoalbuminemia may also occur in familial TTR amyloidosis late in the disease due to autonomic neuropathy-associated malnutrition.

What conclusions might one draw from this study? While the emphasis of the study and its analysis focuses on the different intensity of uptake (or lack thereof) between AL amyloid and TTR-derived amyloid, we would suggest that its main value lies in the potential diagnosis of senile systemic amyloidosis (SSA) [21]. AL amyloidosis is a multisystem disease and can be strongly suspected both from its clinical presentation and abnormal hematologic findings, and familial amyloidosis (caused by a mutant TTR) may be suspected by the frequent coexistence of a polyneuropathy and diagnosed by genetic testing. Unlike other forms of systemic amyloidosis, SSA is, to all intents and purposes, exclusively limited to the heart and presents as heart failure in older men, almost always in their 70s or older. SSA is caused by deposition of amyloid derived from wild-type TTR and is almost certainly a markedly underdiagnosed disease. In a recent survey of types of cardiac amyloidosis seen at two Italian centers, SSA represented only $6.5 \%$ of cases, compared to AL amyloidosis, which represented two thirds of cases [22]. In contrast, in our program which has no age limit for cardiac biopsy and an aggressive approach in underdiagnosed congestive heart failure, over $50 \%$ of the patients with cardiac amyloidosis have TTR amyloid; a figure that persists even after adjusting for potential referral bias by excluding those referred with the amyloid type already known [23].

We believe that the underdiagnosis of SSA is due partly to a failure to consider it in the differential diagnosis of a patient with heart failure and thickened left ventricular walls. However, even if considered, there may be reticence to pursue the diagnosis, largely because it requires endomyocardial biopsy for diagnosis and the patients in whom it occurs are of an age at which cardiologists may express concern about using an invasive technique, particularly if they have a perception that there is no therapy. In view of the recent development of drugs aimed at slowing or stopping the disease, either by stabilizing the precursor TTR and preventing amyloidogenic breakdown products or by decreasing expression of the protein, it becomes all the more important to both heighten awareness of TTR amyloidosis and to make a specific diagnosis [3-5, $7,8]$. The availability of an inexpensive, widely available, and sensitive technique for detecting suspected TTR amyloidosis in an older patient with unexplained heart failure and a nondilated cardiomyopathy is thus both timely and important. A positive ${ }^{99 \mathrm{~m}}$ Tc-DPD cardiac uptake should allow the clinician to focus on those patients with significant uptake and will decrease the need for more invasive techniques in patients at lower risk of this disorder. This simple scan, with its potential to improve the prediction of TTR in elderly patients with unexplained heart failure, thus comes at an opportune time.

\section{References}

1. Falk RH, Dubrey SW. Amyloid heart disease. Prog Cardiovasc Dis 2010;52:347-61.

2. Comenzo RL. How I treat amyloidosis. Blood 2009;114:3147-57.

3. Kolstoe SE, Wood SP. Drug targets for amyloidosis. Biochem Soc Trans 2010;38:466-70.

4. Kolstoe SE, Mangione PP, Bellotti V, Taylor GW, Tennent GA, Deroo S, et al. Trapping of palindromic ligands within native transthyretin prevents amyloid formation. Proc Natl Acad Sci U S A 2010;107:20483-8.

5. Gillmore JD, Tennent GA, Hutchinson WL, Gallimore JR, Lachmann HJ, Goodman HJ, et al. Sustained pharmacological depletion of serum amyloid $\mathrm{P}$ component in patients with systemic amyloidosis. Br J Haematol 2010;148:760-7.

6. Bodin K, Ellmerich S, Kahan MC, Tennent GA, Loesch A, Gilbertson JA, et al. Antibodies to human serum amyloid $\mathrm{P}$ component eliminate visceral amyloid deposits. Nature 2010;468:93-7.

7. Benson MD, Kluve-Beckerman B, Zeldenrust SR, Siesky AM, Bodenmiller DM, Showalter AD, et al. Targeted suppression of an amyloidogenic transthyretin with antisense oligonucleotides. Muscle Nerve 2006;33:609-18.

8. Picken MM. Amyloidosis-where are we now and where are we heading? Arch Pathol Lab Med 2010;134:545-51.

9. Perugini E, Guidalotti PL, Salvi F, Cooke RM, Pettinato C, Riva L, et al. Noninvasive etiologic diagnosis of cardiac amyloidosis using 99mTc-3,3-diphosphono-1,2-propanodicarboxylic acid scintigraphy. J Am Coll Cardiol 2005;46:1076-84.

10. Rapezzi C, Quarta CC, Guidalotti PL, Longhi S, Pettinato C, Leone $\mathrm{O}$, et al. Usefulness and limitations of $99 \mathrm{mTc}-3,3-$ diphosphono-1,2-propanodicarboxylic acid scintigraphy in the aetiological diagnosis of amyloidotic cardiomyopathy. Eur J Nucl Med Mol Imaging 2011. doi:10.1007/s00259-010-1642-7 
11. Glaudemans AW, Slart RH, Zeebregts CJ, Veltman NC, Tio RA, Hazenberg BP, et al. Nuclear imaging in cardiac amyloidosis. Eur J Nucl Med Mol Imaging 2009;36:702-14.

12. Han S, Chong V, Murray T, McDonagh T, Hunter J, Poon FW, et al. Preliminary experience of $99 \mathrm{mTc}$-aprotinin scintigraphy in amyloidosis. Eur J Haematol 2007;79:494-500.

13. Hongo M, Urushibata K, Kai R, Takahashi W, Koizumi T, Uchikawa $\mathrm{S}$, et al. Iodine-123 metaiodobenzylguanidine scintigraphic analysis of myocardial sympathetic innervation in patients with AL (primary) amyloidosis. Am Heart J 2002;144:122-9.

14. Falk RH, Lee VW, Rubinow A, Hood WB, Cohen AS. Sensitivity of technetium-99m-pyrophosphate scintigraphy in diagnosing cardiac amyloidosis. Am J Cardiol 1983;51:826-30.

15. Gertz MA, Brown ML, Hauser MF, Kyle RA. Utility of technetium Tc $99 \mathrm{~m}$ pyrophosphate bone scanning in cardiac amyloidosis. Arch Intern Med 1987;147:1039-44.

16. Hongo M, Hirayama J, Fujii T, Yamada H, Okubo S, Kusama S, et al. Early identification of amyloid heart disease by technetium99m-pyrophosphate scintigraphy: a study with familial amyloid polyneuropathy. Am Heart J 1987;113:654-62.

17. Lee VW, Caldarone AG, Falk RH, Rubinow A, Cohen AS. Amyloidosis of heart and liver: comparison of Tc-99m pyrophos- phate and Tc-99m methylene diphosphonate for detection. Radiology 1983;148:239-42.

18. Maceira AM, Joshi J, Prasad SK, Moon JC, Perugini E, Harding I, et al. Cardiovascular magnetic resonance in cardiac amyloidosis. Circulation 2005; 111:186-93.

19. Pennell DJ, Maceira AM. Magnetic resonance imaging in cardiac amyloidosis. JACC Cardiovasc Imaging 2009;2:137880.

20. Charkes ND, Makler Jr PT. Studies in skeletal tracer kinetics. V: Computer-simulated Tc-99m (Sn)MDP bone-scan changes in some systemic disorders: concise communication. J Nucl Med 1981;22:601-5.

21. Ng B, Connors LH, Davidoff R, Skinner M, Falk RH. Senile systemic amyloidosis presenting with heart failure: a comparison with light chain-associated amyloidosis. Arch Intern Med 2005;165:1425-9.

22. Rapezzi C, Merlini G, Quarta CC, Riva L, Longhi S, Leone O, et al. Systemic cardiac amyloidoses: disease profiles and clinical courses of the 3 main types. Circulation 2009;120:1203-12.

23. Kruger JL, Falk RH. An underdiagnosed disease? High prevalence of senile amyloidosis in a cardiac amyloid program (abstract). Amyloid 2010;17 Suppl 1:207. 